\title{
EHMTI-0228. A clinical interview versus prospective headache diaries in the diagnosis of menstrual migraine without aura
}

\author{
KG Vetvik ${ }^{1 *}$, EA MacGregor ${ }^{2}, A C$ Lundqvist $^{3}, M B$ Russell ${ }^{4}$ \\ From 4th European Headache and Migraine Trust International Congress: EHMTIC 2014 \\ Copenhagen, Denmark. 18-21 September 2014
}

\section{Introduction}

The International Classification of Headache Disorders (ICHD) II and III beta defines menstrual migraine as attacks of migraine without aura occurring on day $1 \pm 2$ of the menstrual cycle in $\geq 2 / 3$ menstruations. According to the ICHD III beta, a three month prospective headache diary is required in order to establish the diagnosis of MM.

\section{Aims}

To compare the diagnosis of MM from a clinical interview based on the ICHD II-criteria for MM, with prospective headache diaries in a population-based study.

\section{Methods}

237 women with self-reported migraine in at least half of menstruations were interviewed by a neurologist about headache and diagnosed according to ICHD II. Additionally, the MM-criteria were expanded to include other types of migraine related to menstruation. Subsequently, all women were asked to complete three month prospective headache diaries.

\section{Results}

A total of 123 (52\%) women completed both clinical interview and diaries. Thirty-eight women were excluded from the analyses; two had incomplete diaries and 36 women recorded $£ 1$ menstruation, leaving 85 diaries eligible for analysis. Sensitivity, specificity, positive and negative predictive value and Kappa for the diagnosis of MM in clinical interview vs. headache diary were $82 \%$, $83 \%, 90 \%, 71 \%$ and 0.62 (95\% CI $0.46-0.80)$. Using a broader definition of MM, Kappa was 0.64 (95\% CI 0.47-0.83).

\section{Conclusion}

A thorough clinical interview is valid for the diagnosis of MM. When this is undertaken, prospective headache diaries should not be mandatory to diagnose MM but may be necessary to exclude a chance association.

No conflict of interest.

\section{Authors' details}

'Institute of Clinical Medicine and Head and Neck Research group Research Centre, University of Oslo and Akershus University Hospital, Lorenskog, Norway. ${ }^{2}$ Barts and the London School of Medicine and Dentistry, Centre for Neuroscience and Trauma Blizard Institute, London, UK. ${ }^{3} \mathrm{Head}$ and Neck Research Group and HØKH Research Centre and Institute of Clinical Medicine and Department of Neurology, Akershus University Hospital and University of Oslo, Lørenskog, Norway. ${ }^{4}$ Head and Neck Research Group Research Centre and Institute of Clinical Medicine, Akershus University Hospital and University of Oslo, Lørenskog, Norway.

Published: 18 September 2014 